\title{
Molecular Cloning and Characterization of Fengycin Synthetase Gene fenB from Bacillus subtilis

\author{
GUANG-HUEY LIN,,${ }^{1,2}$ CHYI-LIANG CHEN, ${ }^{2}$ JOHANNES SCHENG-MING TSCHEN,${ }^{3}$ SAN-SAN TSAY, \\ YU-SUN CHANG, ${ }^{2}$ AND SHIH-TUNG LIU ${ }^{2 *}$ \\ Graduate Institute of Botany, National Taiwan University, Taipei, $106,{ }^{1}$ Graduate Institute of Botany, \\ National Chung-Hsing University, Taichung, 402, ${ }^{3}$ and Molecular Genetics Laboratory, \\ Department of Microbiology and Immunology, Chang-Gung University, \\ Kwei-Shan, Taoyuan, 333, ${ }^{2}$ Taiwan
}

Received 3 November 1997/Accepted 30 December 1997

\begin{abstract}
A fengycin synthetase gene, $f e n B$, has been cloned and sequenced. The protein (FenB) encoded by this gene has a predicted molecular mass of $143.6 \mathrm{kDa}$. This protein was overexpressed in Escherichia coli and was purified to near homogeneity by affinity chromatography. Experimental results indicated that the recombinant FenB has a substrate specificity toward isoleucine with an optimum temperature of $25^{\circ} \mathrm{C}$, an optimum $\mathrm{pH}$ of 4.5, a $K_{m}$ value of $922 \mu \mathrm{M}$, and a turnover number of $236 \mathrm{~s}^{-1}$. FenB also consists of a thioesterase domain, suggesting that this protein may be involved in the activation of the last amino acid of fengycin.
\end{abstract}

Fengycin is a lipopeptidic antifungal antibiotic produced by Bacillus subtilis F29-3 (2, 4), consisting of 10 amino acids and having a primary sequence similar to that of plipastatin $(10,16$, 24). Mutagenesis and sequencing studies found that fengycin is probably synthesized nonribosomally by peptide synthetases $(1,2)$. A peptide synthetase may consist of one to several amino acid activation modules for the activation of specific amino acids (9). In each module, there is an amino acid adenylation domain of approximately 500 amino acids, consisting of five highly conserved motifs for ATP binding and for ATPase activity (19). Mutation in the motifs can significantly reduce the activity of amino acid activation $(6,7)$, indicating that these motifs are indeed essential for peptide synthesis (7). In a peptide synthetase module, the C-terminal boundary of the activation domain is followed by a thioester formation domain which contains a conserved DNFYxLGGHSL motif for the binding of cofactor $4^{\prime}$-phosphopantetheine $(9,19)$. After adenylation, the amino acid is transferred to the $4^{\prime}$-phosphopantetheine at the carrier domain (20). A transpeptidation step subsequently follows, which transfers the amino acid on the cofactor of the initiating module to the activated amino acid at the thioester formation domain in the next module to form a peptide (9). This condensation step continues from one module to the other until a complete peptide is synthesized (9). It is thought that peptide synthetases may form a complex in vivo and the amino acid activation modules among the enzymes are connected and aligned colinearly with the sequence of the amino acids in the antibiotic $(8,18)$, thereby allowing an antibiotic with the correct sequence to be sequentially synthesized. A peptide synthetase also consists of a conserved spacer domain which is present at the $\mathrm{N}$-terminal region, upstream from the adenylation domain of each module (4), except for the module activating the initiating amino acid in which the spacer domain is located in the C-terminal end, downstream from the thioester carrier domain (20). In addition, the $\mathrm{C}$ terminus of the last module of a peptide synthetase may contain an epimerization domain for the conversion of L-amino

\footnotetext{
* Corresponding author. Molecular Genetics Laboratory, Department of Microbiology and Immunology, Chang-Gung University, Kwei-Shan, Taoyuan, 333, Taiwan. Phone: 886-3-328-0292. Fax: 8863-328-0292. E-mail: cgliu@cguaplo.cgu.edu.tw.
}

acid to D-amino acid (4) and a spacer domain which may be essential for the elongation of peptide. The peptide synthetases involved in the activation of the last amino acid of a peptide usually consist of a thioesterase-like domain in the C-terminal region (3). This domain may be responsible for the release of the peptide from $4^{\prime}$-phosphopantetheine, a prerequisite for terminating nonribosomal peptide synthesis (18). In this study, we have cloned, sequenced, and characterized a fengycin synthetase gene, $f e n B$. This gene is involved in the activation of the last amino acid of fengycin.

Nucleotide sequence of $\boldsymbol{f e n B}$. In a previous study (2), we identified a 46-kb cosmid clone, pFC660, which contains genes encoding fengycin synthesis. This cosmid consists of three Bam HI fragments-B1 (18 kb), B2 (12 kb), and B3 (16 kb) (2). In this study, we have sequenced the entire B2 fragment and found that this fragment is actually $11,459 \mathrm{bp}$ long. In the $3^{\prime}$ portion of the fragment, there is a 3,825-bp gene, fen $B$, which is preceded by a ribosomal binding site and is followed by a putative transcriptional stop signal, which consists of a stemloop structure and a stretch of T's. The 5' portion of the B2 fragment, ranging from nucleotides (nt) 1 to 6,036, consists of an incomplete open reading frame, which is actually the $3^{\prime}$ portion of a 10,488-bp peptide synthetase gene, fen $A$. The

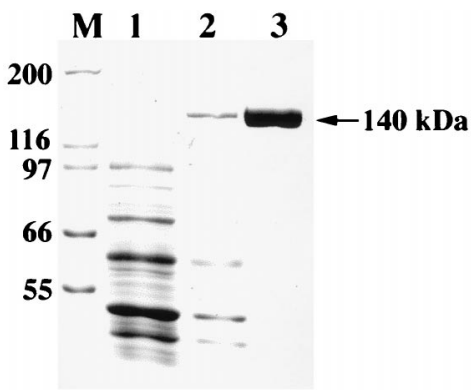

FIG. 1. Expression and purification of His-tagged recombinant FenB. Cell extracts obtained from cells before (lane 1) and after (lane 2) IPTG induction and proteins eluted from His-Bind column (lane 3 ) were analyzed by SDS-PAGE and stained by Coomassie blue. The top band in lane 2 is overexpressed FenB $(140 \mathrm{kDa})$. The positions of molecular mass markers $(\mathrm{M})$ (in kilodaltons) are shown to the left of the gel. 

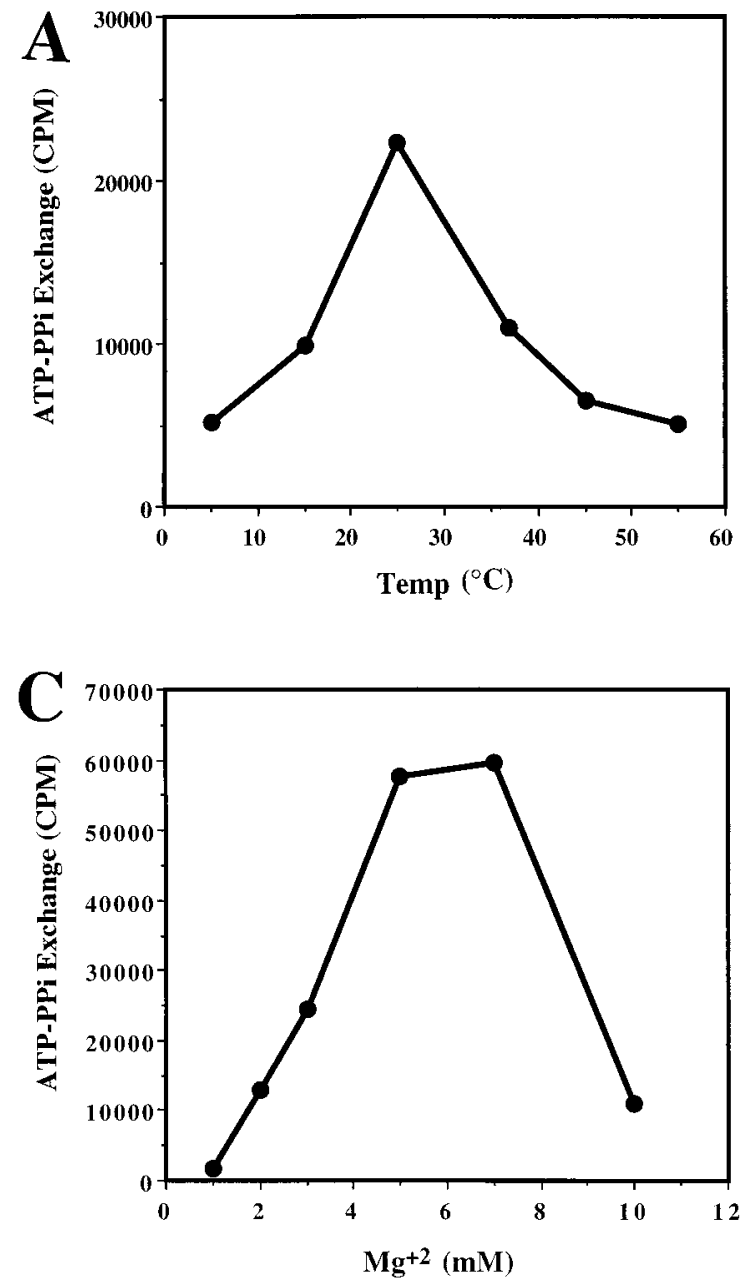

protein encoded by $f e n B(F e n B)$ consists of six core sequences (Table 1) and a thioesterase-like domain (GYSAG) which are highly conserved among peptide synthetases $(3,5)$. The fenB sequence shows $80.6 \%$ homology to a gene in the pps operon of $B$. subtilis 168 (21). Since B. subtilis 168 does not produce fengycin, it is unclear whether the fen $B$-like gene in strain 168 is functional or whether the proteins encoded by these two genes have the same function.

Expression and purification of FenB. To obtain a sufficient amount of FenB for enzyme analysis, we overexpressed fenB in Escherichia coli M15(pRep4) (Qiagen, Hilden, Germany). This overexpression was accomplished by cloning $\mathrm{fen} B$ into an expression vector, pQE60 (Qiagen). The $\operatorname{fen} B$ DNA (nt 1 to 3822) was amplified by using primers B1 (5'-ATCCATGGTT

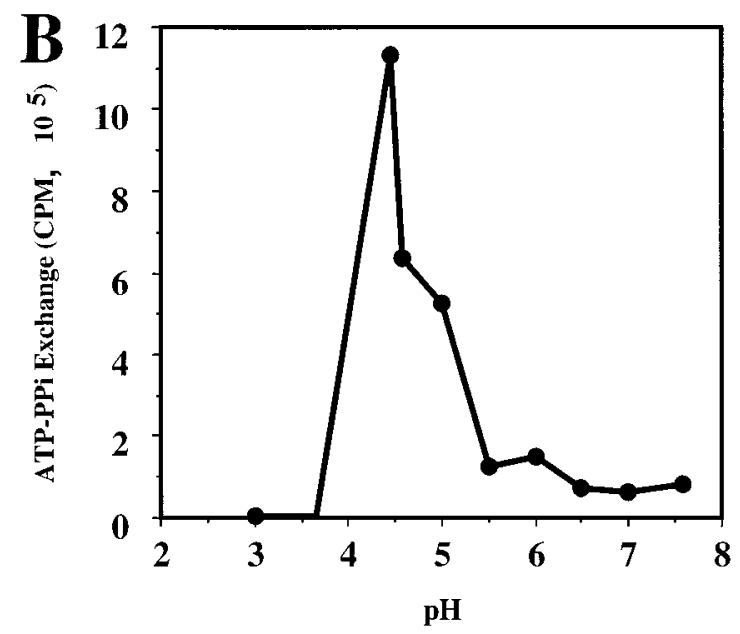

FIG. 2. Properties of FenB. Effects of temperature (A), $\mathrm{pH}(\mathrm{B})$, and $\mathrm{Mg}^{2+}$ concentration (C) in FenB activity. The optimum $\mathrm{Mg}^{2+}$ concentration was determined in a buffer containing 2 mM EDTA.

AAAAACCAAAAAAAT) and B2 (5'-ACGGATCCATGCT TATTTGGCAGC), which contained an NcoI restriction site and a Bam HI restriction site at 5' ends, respectively. PCR was then performed for 30 cycles, with 1 cycle consisting of $1 \mathrm{~min}$ at $94^{\circ} \mathrm{C}, 2 \mathrm{~min}$ at $40^{\circ} \mathrm{C}$, and $3 \mathrm{~min}$ at $72^{\circ} \mathrm{C}$. The amplified fragment was cut by $B a m \mathrm{HI}$ and $N c o$ I and was inserted into the NcoI-BamHI sites of pQE60. FenB expression was induced by isopropyl- $\beta$-D-thiogalactopyranoside (IPTG) treatment. For the purification of FenB, cells were frozen in liquid nitrogen and then were thawed at room temperature. A total of three cycles of freeze-thawing were conducted. Cells were suspended in $4 \mathrm{ml}$ of buffer containing $5 \mathrm{mM}$ imidazole, $0.5 \mathrm{mM} \mathrm{NaCl}$, and $20 \mathrm{mM}$ Tris- $\mathrm{HCl}(\mathrm{pH} 7.9)$ and were sonicated at $0^{\circ} \mathrm{C}$ for 48 5 -s pulses at 10-s intervals with an output control setting at 3 with a sonicator (model UP400A; Ultrasonic Processor Corp., Copiague, N.Y.). Next, cell extract was centrifuged at 15,000 rpm for $60 \mathrm{~min}$ at $4^{\circ} \mathrm{C}$ with a Sorvall SS-34 rotor. FenB in the supernatant was then purified with a His-Bind column (Novagen, Madison, Wis.) (1.5 by $4 \mathrm{~cm})$, and FenB in the fractions was examined by sodium dodecyl sulfate-polyacrylamide gel electrophoresis (SDS-PAGE) (12) and by staining with Coomassie blue (Merck, Darmstadt, Germany). The expressed FenB has a molecular mass of $140 \mathrm{kDa}$, as determined by SDS-PAGE (Fig. 1, lanes 2 and 3). The chromatography procedure was able to purify FenB to near homogeneity (Fig. 1, lane 3). In addition, approximately $300 \mu \mathrm{g}$ of recombinant FenB could be purified from $50 \mathrm{ml}$ of culture.

Substrate specificity. The enzymatic activity of recombinant FenB was determined by an ATP-PP $\mathrm{i}_{\mathrm{i}}$ exchange assay (14) using a reaction mixture containing $\left[{ }^{32} \mathrm{P}\right]$ tetrasodium pyro-

TABLE 1. Comparison of the amino acid sequences conserved in peptide synthetases and FenB

\begin{tabular}{|c|c|c|c|c|}
\hline Motif $^{a}$ & Conserved sequence $^{a}$ & Function $^{a}$ & Sequence in FenB & Positions in $\operatorname{fen} B$ (nt) \\
\hline Spacer & HHILxDGW & Unknown & HHILMDGGW & $435-459$ \\
\hline Core 1 & LKAGGAYVPID & Unknown & LKAGGTYLPLD & $1,617-1,650$ \\
\hline Core 2 & YSGTTGxPKGV & ATP binding & SSGSTGRPKGV & $1,851-1,881$ \\
\hline Core 3 & GELCIGGxGxARGYL & ATP binding & GELCVGGEGVAKGYL & $2,409-2,454$ \\
\hline Core 4 & YxTGD & ATPase & YRTGD & $2,517-2,532$ \\
\hline Core 5 & VKIRGxRIELGEIE & ATP binding & IKIRGKRIEPAEIE & $2,559-2,631$ \\
\hline Core 6 & DNFYxLGGHSL & $\begin{array}{l}\text { 4'-Phosphopantetheine binding } \\
\text { (thioester formation) }\end{array}$ & DFFALGGHSL & $2,982-3,012$ \\
\hline
\end{tabular}

\footnotetext{
${ }^{a}$ Data taken from Stachelhaus and Marahiel $(18,19)$.
} 
TABLE 2. ATP-PP ${ }_{i}$ exchange activity of FenB

\begin{tabular}{lcc}
\hline Amino acid & Exchange activity $(\mathrm{cpm})^{a}$ & $\%$ Activity \\
\hline Isoleucine & 107,038 & 100 \\
Glutamic acid & 284 & 0.26 \\
Alanine & 3,370 & 3.1 \\
Proline & 1,384 & 1.2 \\
Ornithine & 1,582 & 1.4 \\
Threonine & 2,171 & 2.0 \\
Tyrosine & 1,834 & 1.7 \\
Valine & 13,630 & 12.7 \\
Blank & 1,497 & 1.4 \\
\hline
\end{tabular}

${ }^{a}$ The reaction mixture consisted of $84 \mu \mathrm{g}$ of recombinant FenB. The reaction was allowed to proceed for $10 \mathrm{~min}$.

phosphate (9,120 Ci/mmol) (NEN, Boston, Mass.), $2 \mathrm{mM}$ ATP, and $2 \mathrm{mM}$ amino acid. The amino acids used for the assay included the eight amino acids present in the fengycin molecule (Table 2) $(10,25)$ as well as other common amino acids. Experimental results indicated that adding isoleucine to the reaction mixture produced the highest ATP-PP $\mathrm{P}_{\mathrm{i}}$ exchange activity (Table 2), i.e., approximately 8 - to 70 -fold higher than the binding to the other amino acids. Above results suggested that FenB has a substrate specificity toward isoleucine. Previous reports have demonstrated that isoleucine is the last amino acid of the fengycin molecule $(10,25)$. This finding suggests that FenB is not only responsible for activating the last amino acid of fengycin but also involved in releasing the fengycin molecule from the peptide synthetase. Actually, the amino acid sequence of FenB also reveals that this is indeed the case. In the C-terminal region of FenB, the protein consists of a thioesterase-like domain instead of an epimerase domain, a feature shared by all the peptide synthetases involved in activating the last amino acid of antibiotics $(3,5)$.

Binding of amino acid to FenB. Covalent binding of amino acid to FenB was examined with $1 \mu \mathrm{Ci}$ of $\mathrm{L}-\left[{ }^{14} \mathrm{C}\right]$ isoleucine (315 mCi/mmol) (Amersham, Buckinghamshire, England) and $11 \mu \mathrm{g}$ of purified recombinant FenB by the method described by Ullrich et al. (23). The reaction was allowed to proceed for $30 \mathrm{~min}$ at $37^{\circ} \mathrm{C}$ and then was stopped by adding $2 \mathrm{ml}$ of ice-cold $10 \%$ trichloroacetic acid. Our results demonstrated that $\left[{ }^{14} \mathrm{C}\right]-$ isoleucine could bind to FenB covalently and gave a radioactivity reading of $6,725 \mathrm{cpm}$, whereas the negative control, which lacked ATP in the reaction mixture, had a value of 98 $\mathrm{cpm}$. This binding is specific, since nonradioactive isoleucine, when added in an excessive amount in the reaction mixture, could compete with the binding of radioactive isoleucine to FenB. In theory, binding of isoleucine to FenB requires a prior binding of $4^{\prime}$-phosphopantetheine to the enzyme $(11,17)$. In the case of surfactin synthetases, this binding is catalyzed by the enzyme encoded by the $s f p$ gene in B. subtilis (13). A similar gene is also involved in plipastatin synthesis (22). A previous study has demonstrated that approximately $14 \%$ of the peptide synthetase expressed in $E$. coli has a phosphopantetheinyl group attached to the enzyme (19). This binding is catalyzed by an $E$. coli enzyme, phosphopantetheinyl transferase $(17,19)$. Presumably, the phosphopantetheinyl group of coenzyme A is transferred to FenB by the same mechanism and subsequently results in the binding of the $\left[{ }^{14} \mathrm{C}\right]$ isoleucine to the enzyme. We found that approximately $13 \%$ of FenB expressed in E. coli bound to the amino acid.

Biochemical characterization of recombinant FenB. The recombinant FenB enzyme had optimum activity at $25^{\circ} \mathrm{C}$ (Fig. 2A), at $\mathrm{pH} 4.5$ (Fig. 2B), and with a $\mathrm{Mg}^{2+}$ concentration between 5 and $8 \mathrm{mM}$ in a buffer containing 2 mM EDTA (Fig.
2C). The activity of FenB at $\mathrm{pH} 7.0$ is approximately 18 -fold lower than the activity exhibited under $\mathrm{pH} 4.5$ (Fig. 2B). The low optimum $\mathrm{pH}$ for FenB may allow the enzyme to function efficiently in the acidic intracellular environment. Although many peptide synthetases have been isolated from Bacillus spp. and characterized biochemically $(11,15,19)$, the optimum $\mathrm{pHs}$ of these enzymes were not determined in those studies. The activity also decreased when the $\mathrm{Mg}^{2+}$ concentration exceeded $10 \mathrm{mM}$ (Fig. 2C). It is likely that a high concentration of $\mathrm{Mg}^{2+}$ affects the amount of EDTA, which may be critical in maintaining the enzyme's stability. The recombinant FenB, under optimum conditions, exhibited Michaelis-Menten kinetics, with a $K_{m}$ for isoleucine of $922 \mu \mathrm{M}$ and a turnover number of $236 \mathrm{~s}^{-1}$.

In summary, we have sequenced and characterized the fengycin synthetase gene $\operatorname{fen} B$ from $B$. subtilis F29-3. Experimental results demonstrate that the FenB protein functions as a peptide synthetase which is involved in the nonribosomal synthesis of fengycin. This enzyme is responsible for the adenylation of isoleucine and for the binding of the amino acid to its cofactor, 4'-phosphopantetheine. Evidence presented herein suggests that FenB is involved in the activation of the last amino acid of the fengycin peptide. Our results should provide a valuable reference for future studies involving fengycin synthesis.

Nucleotide sequence accession numbers. The nucleotide sequences of the 3,825-bp fen $B$ gene and the 10,488-bp fen $A$ gene have been deposited in GenBank under accession no. L42523 and AF023464, respectively.

We thank Hans von Döhren and J.-S. Yu for their technical advice. This research was supported by Medical Research Grant CMRP525 from the Chang-Gung Memorial Hospital and by Biological Research Grant NSC-86-2314-B-182-028 from the National Science Council of the Republic of China.

\section{REFERENCES}

1. Chang, L. K., C. L. Chen, Y. S. Chang, J. S. M. Tschen, J. M. Chen, and S. T. Liu. 1994. Construction of Tn917ac1, a transposon useful for mutagenesis and cloning of Bacillus subtilis genes. Gene 150:129-134.

2. Chen, C. L., L. K. Chang, Y. S. Chang, S.-T. Liu, and J. S. M. Tschen. 1995. Transposon mutagenesis and cloning of the genes encoding the enzymes of fengycin biosynthesis in Bacillus subtilis. Mol. Gen. Genet. 243:121-125.

3. Cosmina, P., F. Rodriguez, F. de Ferra, G. Grandi, M. Perego, G. Venema, and D. von Sinderen. 1993. Sequence and analysis of the genetic locus responsible for surfactin synthesis in Bacillus subtilis. Mol. Microbiol. 8:821831.

4. de Crécy-Lagard, V., P. Marliere, and W. Saurin. 1995. Multienzymatic nonribosomal peptide biosynthesis: identification of the functional domains catalyzing peptide elongation and epimerization. Life Sci. 318:927-936.

5. de Ferra, F., F. Rodriguez, F. Tortora, C. Tosi, and G. Grandi. 1997. Engineering of peptide synthetase. J. Biol. Chem. 272:25304-25309.

6. Elsner, A., H. Enger, W. Saenger, L. Hamoen, G. Venema, and F. Bernhard. 1997. Substrate specificity of hybrid modules from peptide synthetases. J. Biol. Chem. 272:4814-4819.

7. Gocht, M., and M. A. Marahiel. 1994. Analysis of core sequences in the D-phe activating domain of the multifunctional peptide synthetase TycA by site-directed mutagenesis. J. Bacteriol. 176:2654-2662.

8. Kleinkauf, H., and H. von Döhern. 1990. Nonribosomal biosynthesis of peptide antibiotics. Eur. J. Biochem. 192:1-15.

9. Kleinkauf, H., and H. von Döhren. 1996. A nonribosomal system of peptide biosynthesis. Eur. J. Biochem. 236:335-351.

10. Koch, U. 1988. Fengycin: Strukturaufklärung eines mikroheterogene Lipopeptidolidantibiotikums. Dissertation, zur Erlangung des Grades eines Doktors, der Naturwissenschaften. Eberhard-Karls-Universität zu Tübingen, Tübingen, Germany.

11. Ku, J., R. G. Mirmira, L. Liu, and D. L. Santi. 1997. Expression of a functional non-ribosomal peptide synthetase module in Escherichia coli by coexpression with a phosphopantetheinyl transferase. Chem. Biol. 4:203207.

12. Laemmli, U. K. 1970. Cleavage of structural proteins during the assembly of the head of bacteriophage T4. Nature 227:680-685.

13. Lambalot, R. H., A. M. Gehring, R. S. Flugel, P. Zuber, M. LaCelle, M. A. Marachiel, R. Reid, C. Khosla, and C. T. Walsh. 1996. A new enzyme 
superfamily - the phosphopantetheinyl transferases. Chem. Biol. 3:923-936.

14. Lee, S. G., and F. Lipmann. 1975. Tyrocidine synthetase. Methods Enzymol. 43:585-602.

15. Mootz, H., and M. A. Maraheil. 1997. The tyrocidine biosynthesis operon of Bacillus brevis: complete nucleotide sequence and biochemical characterization of functional internal adenylation domains. J. Bacteriol. 179:6843-6850.

16. Nishikiori, T., H. Naganawa, Y. Muraoka, T. Aoyagi, and H. Umezawa. 1986. Plipastatins: new inhibitors of phospholipase A2, produced by Bacillus cereus BMG302-fF67. III. Structural elucidation of plipastatins. J. Antibiot. 39:755761.

17. Stachelhaus, T., A. Hüser, and M. A. Marahiel. 1996. Biochemical characterization of peptidyl carrier protein (PCP), the thiolation domain of multifunctional peptide synthetases. Chem. Biol. 3:913-921.

18. Stachelhaus, T., and M. A. Marahiel. 1995. Modular structure of gene encoding multifunctional peptide synthetase required for non-ribosomal peptide synthesis. FEMS Microbiol. Lett. 125:3-14.

19. Stachelhaus, T., and M. A. Marahiel. 1995. Modular structure of peptide synthetase revealed by dissection of the multifunctional enzyme GrsA. J. Biol. Chem. 270:6163-6169.

20. Stein, T., J. Vater, V. Kruft, A. Otto, B. Wittmann-Liebold, P. Franke, M.
Panico, R. McDowell, and H. R. Morris. 1996. The multiple carrier model of nonribosomal peptide biosynthesis at modular multienzyme templates. J. Biol. Chem. 271:15428-15435.

21. Tognoni, A., E. Franchi, C. Magistrelli, E. Colombo, P. Cosmina, and G. Grandi. 1995. A putative new peptide synthase operon in Bacillus subtilis: partial characterization. Microbiology 141:645-648.

22. Tsuge, K., T. Ano, and M. Shoda. 1996. Isolation of a gene essential for biosynthesis of the lipopeptide antibiotic plipastatin B1 and surfactin in Bacillus subtilis YB8. Arch. Microbiol. 165:243-251.

23. Ullrich, C., B. Kluge, Z. Palacz, and J. Vater. 1991. Cell-free biosynthesis of surfactin, a cyclic lipopeptide produced by Bacillus subtilis. Biochemistry 30:6503-6508.

24. Umezawa, H., T. Aoyagi, T. Nishikori, A. Okuyama, Y. Yamagishi, M. Hamada, and T. Takeuchi. 1986. Plipastatins: new inhibitors of phospholipase A2, produced by Bacillus cereus BMG302-fF67. I. Taxonomy, production and preliminary characterization. J. Antibiot. 39:737-744.

25. Vanittanakom, N., and W. Loeffler. 1986. Fengycin-a novel antifungal lipopeptide antibiotic produced by Bacillus subtilis F29-3. J. Antibiot. 39:888901. 